\title{
Corynebacterium striatum Cardiac Device-Related Infective Endocarditis: The First Case Report in a Patient With a Cardiac Resynchronization Therapy Defibrillator Device and Review of the Literature
}

\author{
Luisa Serpa Pinto ${ }^{a}$,, Andre Dias Frias ${ }^{\mathrm{b}}$, Margarida Franca ${ }^{\mathrm{a}}$
}

\begin{abstract}
Corynebacterium striatum (C. striatum) is a skin commensal agent, rarely described as a cause of infective endocarditis. We describe a case of a 48-year-old man, with multiple comorbidities with cardiac resynchronization therapy defibrillator (CRT-D) device implanted 1 year before. A cardiac device-related infective endocarditis (CDRIE) due to $C$. striatum, with vegetations in the tricuspid valve adjacent to the electrode lead and concomitant lumbar spondylodiscitis were diagnosed. The patient was treated initially with a 6-week course of vancomycin with sterile blood cultures and reduction of inflammatory parameters. Surgery was refused at this stage. Six weeks later, he was readmitted due to $C$. striatum bacteriemia recurrence, with vegetations adhering to the electrode wire, being treated with daptomycin $10 \mathrm{mg} / \mathrm{kg}$ body weight, after presenting renal toxicity to vancomycin. CRT-D device was removed with implantation of epicardial cardiac resynchronization therapy pacemaker (CRT-P). To our knowledge, this might be the first description of $C$. striatum CDRIE in a patient with a CRT-D. In the five cases described in the literature of CDRIE by this agent, early removal of the pacemaker was performed with good results. In this case, the device was removed only after failure of medical treatment alone.
\end{abstract}

Keywords: Infective endocarditis; Corynebacterium striatum; Cardiac device-related infective endocarditis; Cardiac resynchronization therapy defibrillator; Implantable cardioverter defibrillator; Daptomycin

\section{Introduction}

Corynebacterium striatum (C. striatum) is an aerobic and op-

Manuscript submitted November 4, 2020, accepted November 11, 2020

Published online December 30, 2020

${ }^{a}$ Internal Medicine Department, Centro Hospitalar e Universitario do Porto, Porto, Portugal

${ }^{\mathrm{b}}$ Cardiology Department, Centro Hospitalar e Universitario do Porto, Porto, Portugal

${ }^{\mathrm{c} C}$ Corresponding Author: Luisa Serpa Pinto, Internal Medicine Department, Centro Hospitalar e Universitario do Porto, Largo do Prof. Abel Salazar, 4099001 Porto, Portugal. Email: luisaserpapinto@gmail.com

doi: https://doi.org/10.14740/jmc3618 tional anaerobic gram-positive bacillus, commensal from the skin. In the last decades C. striatum is becoming an emerging agent, a multidrug-resistant pathogen with the capacity to form biofilms that can cause infection of endovascular devices [1].

Cardiac device-related infective endocarditis (CDRIE) is defined as an infection extending to the electrode leads, cardiac valve leaflets, or endocardial surface that can result either from pocket infection extending to intracardiac electrodes, or any portion of the device may become infected due to secondary bacteremia resulting in the formation of vegetations. The latter can be found anywhere from the insertion vein to the superior vena cava, on the lead, tricuspid valve, in the right atrial or ventricular endocardium [2]. The presence of infective material along the lead course may not provide typical vegetations of measurable size so that normal transthoracic echocardiography (TTE) and transesophageal echocardiography (TEE) do not rule out CDRIE [2]. Treatment includes both prolonged antibiotic therapy associated with complete hardware removal [2]. Corynebacterium spp. is rarely identified as a pathogen in CDRIE infection [2]. A literature search in PubMed revealed only five case reports of C. striatum CDRIE [3-7]. Although an increased risk of CDRIE has been described in patients with implantable cardioverter-defibrillators compared with permanent pacemakers [2], all cases described in the literature refer to pacemaker patients, this case being the first case description of CDRIE by C. striatum in a cardiac resynchronization therapy defibrillator (CRT-D) patient. On the other hand, in all cases described in the literature [3-7], the pacemaker was removed in the first inpatient days, with a good outcome despite the patient's age and comorbidities. In this case, due to the patient's poor performance status and comorbidities, the surgical intervention was refused at first. The patient was managed with medical treatment only, but despite clinical improvement, he had recurrence of the infection a few weeks later. The device was removed only on the second CDRIE by the same agent with a good outcome despite the patient's overall condition.

\section{Case Report}

We present the case of a diabetic 48-year-old man with nonischemic dilated cardiomyopathy, associated with heart failure 


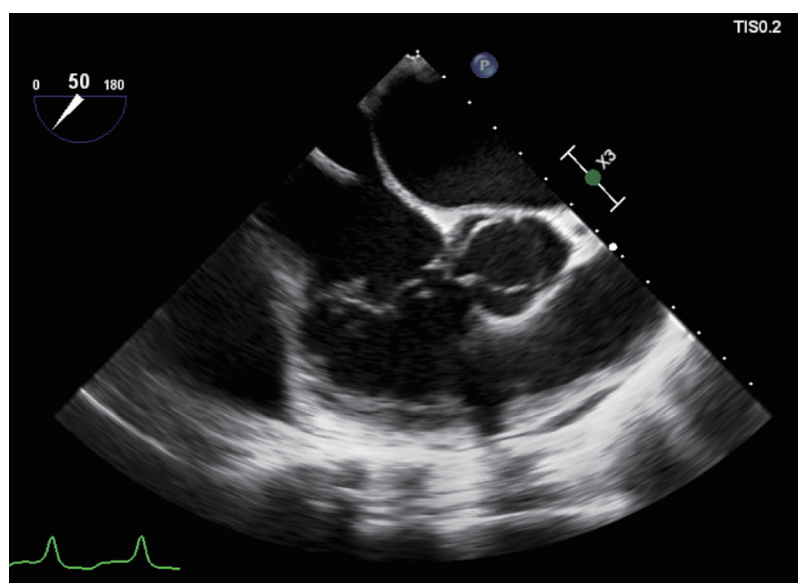

Figure 1. Transesophageal echocardiogram showing the vegetation adherent to the posterior leaflet of tricuspid valve. This exam was unremarkable for fibrin-sheath, masses or vegetations adherent to the electrocatheter.

with severely reduced ejection fraction, complete heart block (CHB), and permanent atrial fibrillation. The patient had a past cardioembolic stroke with left hemiparesis and had a record of recurrent urinary tract infections (UTIs) associated with suprapubic cystostomy due to urethral stenosis. A CRT-D generator (Quadra Assura MP St Jude Medical) was implanted the year before due to $\mathrm{CHB}$ with a single lead pacing cardioverterdefibrillator implanted (ICD), due to QRS $<120 \mathrm{~ms}$ and high probability of widening in the near future.

One month earlier he was admitted in the emergency department (ED) for fever and low back pain, being discharged with a UTI diagnosis and ciprofloxacin.

Again, he was admitted in the ED with fever, severe low back pain, anorexia, and weight loss. On physical examination, fever $\left(39.1^{\circ} \mathrm{C}\right)$, painful palpation of the lumbar spinal apophysis, and absent cardiac audible murmur, or cutaneous embolic phenomena were remarked. Electrocardiography showed atrial fibrillation with complete left bundle branch block with QRS $>150 \mathrm{~ms}$, and TTE was unremarkable for the presence of vegetations. Prior blood cultures (BC) identified C. striatum, with sensitivity to tetracycline and vancomycin, and resistance to gentamicin, clindamycin, ciprofloxacin, and penicillin G; so did a new pair of $\mathrm{BC}$ with the same sensitivity profile. Vancomycin therapy was initiated. $\mathrm{BC}$ collected on the second day of antibiotic was sterile. Ten days after admission TEE showed vegetation at the auricular face of the tricuspid valve, in the posterior leaflet, $+/-6 \mathrm{~mm}$, heterogeneous contours, with two mobile projections with $+/-6 \mathrm{~mm}$, with no apparent device lead involvement (Fig. 1).

Lumbar computed tomography (CT) scan showed irregularities in the L1 - L2 vertebral platforms, which were absent in the previous examination, 1 month earlier. Due to the incompatibility of the CRT-D device, it was not possible to perform magnetic resonance imaging (MRI). However, a lumbar spondylodiscitis diagnosis was presumed.

TEE was repeated (day 29) in the fourth week of vancomycin therapy, similar to the previous one namely regarding the presence of vegetation. Surgical removal of the CRT-D de-

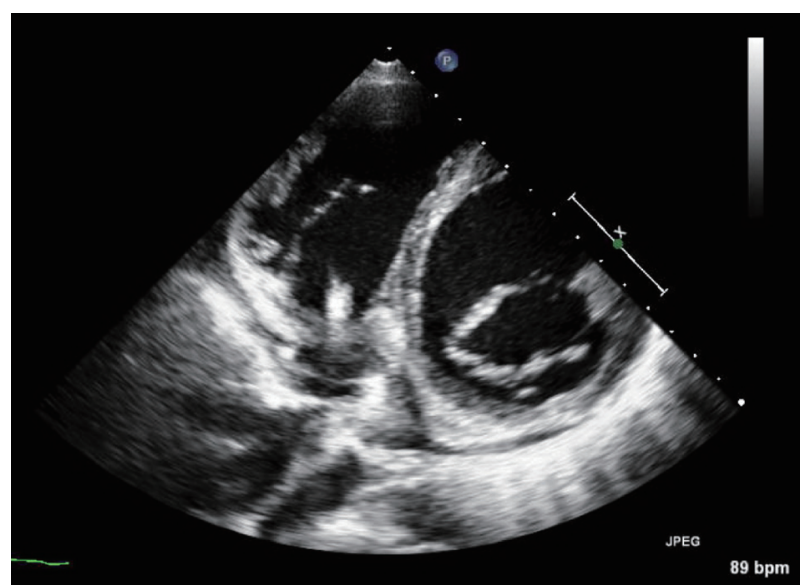

Figure 2. Parasternal short-axis mitral valve unconventional view displaying de novo fibrin-sheath involving the electrocatheter with an additional punctiform image.

vice was proposed, but the patient was considered as having a very high overall surgical risk. So he was managed with medical treatment alone. He was discharged after completing 49 days of vancomycin, with clinical resolution and serial negative blood cultures.

Again, 42 days after hospital discharge, the patient was readmitted, in cardiogenic shock, with respiratory failure, acute kidney injury, and ischemic hepatitis requiring dobutamine. As soon as dysfunctions improved he was transferred to our nursery. Again, BC showed the presence of $C$. striatum, with the same sensitivity profile and presumptively sensitive to daptomycin (minimum inhibitory concentration $(\mathrm{MIC})=0.023 \mu \mathrm{g} / \mathrm{mL}$ ). Neither TTE at admission nor TEE performed 5 days after, showed cardiac vegetation. Vancomycin was started (day 3), requiring several adjustments due to frequent toxic levels. The patient developed acute kidney injury (day 12) suspecting renal toxicity due to vancomycin; and therapy was changed to daptomycin $10 \mathrm{mg} / \mathrm{kg}$. A systolic murmur not present at admission was noticed (day12), and TTE was repeated with evidence of vegetations adhering to the device lead in its auricular path near the tricuspid valve (Fig. 2). Daptomycin therapy was continued without new febrile events or elevated inflammatory markers, and with sterile serial BC. On the 81st day of daptomycin (inpatient day 93), the patient underwent surgical intervention with the removal of CRT-D and implantation of a cardiac resynchronization therapy pacemaker (CRT-P) (Quadra Allure MPP St Jude Medical), with epicardial electrodes and the device placed in an abdominal loca. ICD was not implanted due to the absence of therapies in the past, in addition to frailty and life expectancy $<1$ year. Electrode tips were sterile. He completed 15 days of daptomycin after the intervention. No evidence of recurrence of infection was found in follow-up consultation 6 months after discharge.

\section{Discussion}

Although C. striatum is commensal from the skin, it is becom- 


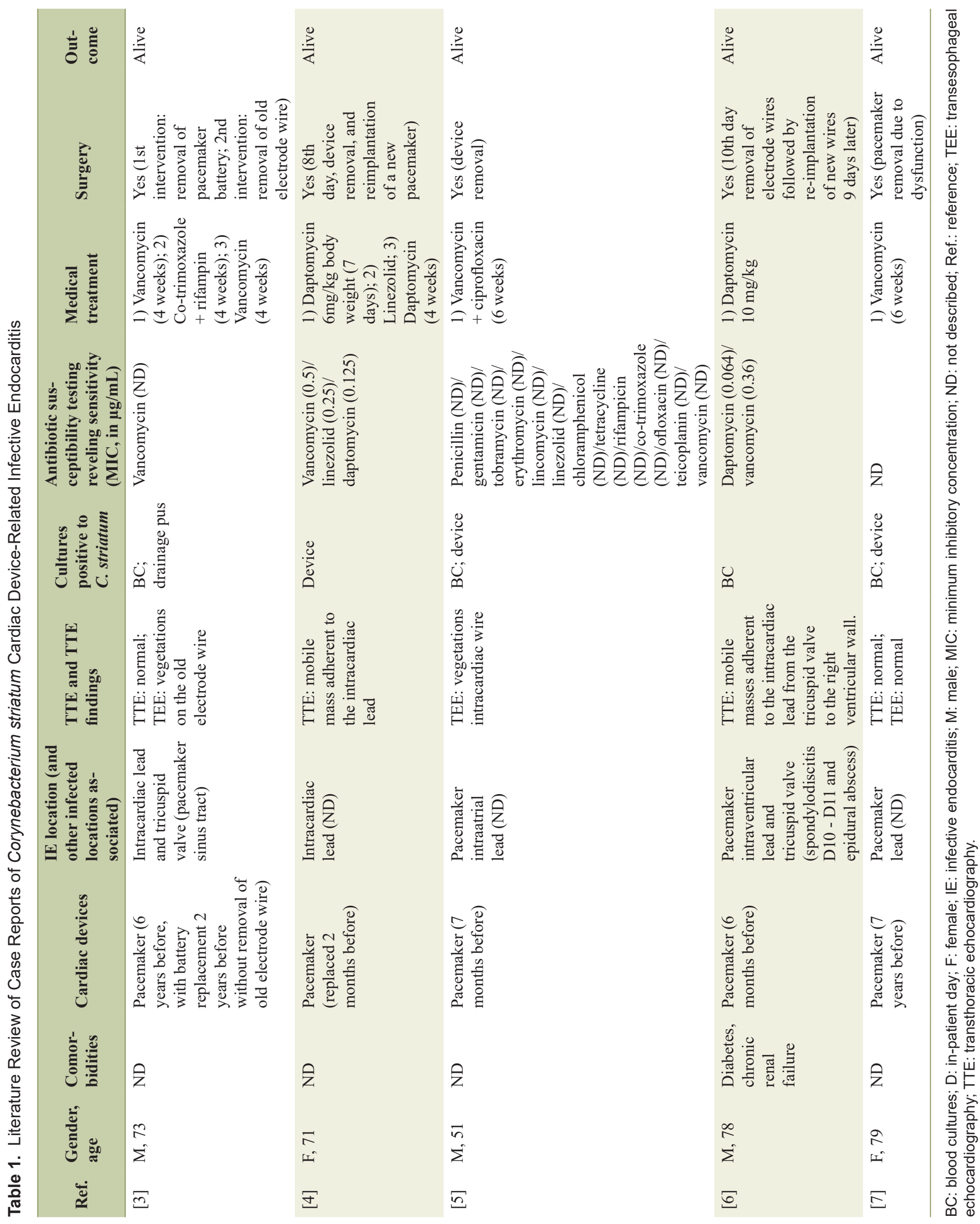


ing an emerging infectious agent, a multidrug-resistant pathogen with the capacity to form biofilms [1]. In the presence of bacteriemia and cardiac devices, CDRIE should be suspected even in the absence of typical vegetations in the TTE and TEE [2]. The treatment combines prolonged antibiotic therapy and device removal [2].

Corynebacterium spp. is rarely identified as a pathogen in infective endocarditis [2], and there are only five case reports of $C$. striatum CDRIE whose characteristics are summarized in Table 1 [3-7]. Most of them (4/5) were treated with vancomycin and all of them with device removal within the first inpatient days. In the case of our patient, despite the assumption of resolution of the bacteremia, clinical improvement, and a 6 weeks course of antibiotics, on the first hospitalization, the infection was not resolved with medical treatment alone with recurrence of bacteremia imposing the withdrawal of CRT-D despite the high surgical risk.

Regarding medical treatment, daptomycin $10 \mathrm{mg} / \mathrm{kg}$ body weight for 6 weeks was successfully used in one case described by Guerrero et al [6], in which spondylodiscitis also coexisted, as in the case of our patient.

It should be noted that only in one of the described cases, vegetations were detected in the TTE, being the TEE necessary to confirm the diagnosis in most cases. Since $C$. striatum is a pathogen capable of forming biofilms, in the presence of bacteremia and endovascular devices, the suspicion for infection should be high and the diagnosis must be pursued, as shown by Szymanska et al [7].

This case demonstrates that in the presence of CDRIE the removal of the device is fundamental to treatment's success and must be pursued to control infection source. On the other hand, it shows that daptomycin is a suitable option for the treatment of infective endocarditis by this pathogen.

\section{Acknowledgments}

None to declare.

\section{Financial Disclosure}

None to declare.

\section{Conflict of Interest}

None to declare.

\section{Informed Consent}

Both written and verbal informed consents were obtained from the patient for publication of this case report.

\section{Author Contributions}

LSP collected the case data, wrote the manuscript, and prepared the table; ADF collected the case data, prepared the pictures, and wrote the manuscript; MF collected the case data and revised the manuscript. All authors approved the final version to be published.

\section{Data Availability}

The authors declare that data supporting the findings of this study are available within the article.

\section{References}

1. McMullen AR, Anderson N, Wallace MA, Shupe A, Burnham CA. When good bugs go bad: epidemiology and antimicrobial resistance profiles of Corynebacterium striatum, an emerging multidrug-resistant, opportunistic pathogen. Antimicrob Agents Chemother. 2017;61(11):e01111-17.

2. Habib G, Lancellotti P, Antunes MJ, Bongiorni MG, Casalta JP, Del Zotti F, Dulgheru R, et al. 2015 ESC Guidelines for the management of infective endocarditis: The Task Force for the Management of Infective Endocarditis of the European Society of Cardiology (ESC). Endorsed by: European Association for Cardio-Thoracic Surgery (EACTS), the European Association of Nuclear Medicine (EANM). Eur Heart J. 2015;36(44):3075-3128.

3. Melero-Bascones M, Munoz P, Rodriguez-Creixems M, Bouza E. Corynebacterium striatum: an undescribed agent of pacemaker-related endocarditis. Clin Infect Dis. 1996;22(3):576-577.

4. Oliva A, Belvisi V, Iannetta M, Andreoni C, Mascellino MT, Lichtner M, Vullo V, et al. Pacemaker lead endocarditis due to multidrug-resistant Corynebacterium striatum detected with sonication of the device. J Clin Microbiol. 2010;48(12):4669-4671.

5. Abi R, Ez-Zahraouii K, Ghazouani M, Zohoun A, Kheyi J, Chaib A, Elouennass M. [A Corynebacterium striatum endocarditis on a carrier of pacemaker]. Ann Biol Clin (Paris). 2012;70(3):329-331.

6. Fernandez Guerrero ML, Molins A, Rey M, Romero J, Gadea I. Multidrug-resistant Corynebacterium striatum endocarditis successfully treated with daptomycin. Int J Antimicrob Agents. 2012;40(4):373-374.

7. Szymanska UA, Kobylecka M, Rosiak M, Ziemba A, Krolicki L, Kosior DA. All that glitters is not gold: positron emission tomography/computed tomography detection failure in a patient with implanted pacemaker and relapsing Corynebacterium striatum sepsis. Kardiol Pol. 2018;76(12):1741. 